\title{
Toward A Theory of Institutional Ecology: The Dynamics of Macro Structural Space
}

\author{
Seth Abrutyn ${ }^{1}$ \\ ${ }^{1}$ Department of Sociology, The University of Memphis, Memphis, TN, USA \\ Correspondence: Seth Abrutyn, Department of Sociology, The University of Memphis, Memphis, 231 Clement \\ Hall, Memphis, TN 38152-0001, USA. Tel: 1-901-678-3031. E-mail: seth.abrutyn@memphis.edu
}

Received: June 14, 2012 Accepted: November 8, 2012 Online Published: November 26, 2012

doi:10.5539/res.v4n5p167 URL: http://dx.doi.org/10.5539/res.v4n5p167

\begin{abstract}
Institutions, like populations and organizations, occupy physical, temporal, symbolic, and social space. It is reasonable, then, to assume institutions are characterized by ecological dynamics. In the paper below, a formal theory of institutional ecology is posited. It is argued that institutions have cores and surrounding environments, and a rule of proximity governs the way individual and corporate actors orient their behavior and attitudes. Hence, the closer are an individual, corporate unit, or field of actors to an institution's core, the greater is the visibility and access to institutional resources and authority systems and, therefore, the more likely they are to act, set goals, and make decisions as sanctioned by the institutional domain. Ultimately, a theory of institutional ecology can help link the micro- and meso-levels while also serving as an explanatory tool for understanding why some actions and goals are stereotyped, while others appear deviant or, in some cases, random.
\end{abstract}

Keywords: institutions, institutional analysis, ecology, sociological theory, macrosociology, organizations

\section{Introduction}

Individuals and corporate actors overwhelmingly act in predictable ways, despite the fact that most actors have the ability to choose from an assortment of lines of action and goals to set. They may or may not share the exact or even proximate meanings or understandings for their convergent behavior or goal setting, nor should we infer the reasons they act as they may be unconscious habits or self-aware strategies designed to fool others, cause 'misrecognition', and for personal gain (Goffman, 1959; Bourdieu, 1977). Over the last thirty years, in reaction to the mistakes made by functionalists and the Parsonsian (1951) systems theory, sociological explanations of behavioral conformity have tended towards one of two directions. Not surprisingly, the social psychological programs like Identity Theory (Stets, 2006) or Affect Control Theory (Robinson and Smith-Lovin, 2006) treat larger forces as exogenous and taken for granted (and vaguely defined), as their interests are in the micro dynamics of identity formation and reproduction within the confines of interactions.

The other direction in sociology has been towards examining meso-level corporate units and the "field" (DiMaggio \& Powell, 1983), niche (Hannan \& Freeman, 1977), or "market" (Fligstein, 1996) comprised of like corporate units struggling for some scarce resources and adopting similar organizational forms and strategies because of convergent forces because of vague pressures like uncertainty or normativity. Thus, like their social psychological counterparts, the meso-level theorists maintain the contemporary bias of bottom-up theorizing which treats the macro-level of analysis as an amorphous environment filled with ambiguous forces and ill-defined dynamics (see Abrutyn \& Turner, 2011 for a review). A careful analysis of these meso-level models reveals a strain towards the macro-level and a desire to understand and explain organizational dynamics in light of much larger forces. For instance, Fligstein and McAdam's (2011) synthetic effort criticizes the various "field" metaphors as conceptualizing a field as an autonomous bounded space, seemingly immune from larger forces. As a first step towards considering the macro-level, Fligstein and McAdam introduce two new concepts: "broader field environments" and "exogenous shocks." However, these two concepts are far older than Fligstein and McAdam give credit and, when considered from a macro-institutional perspective, offer a link between the macro- and meso-levels of social life.

The paper below argues that the neo-evolutionary institutional school (Turner, 2003; cf. Abrutyn \& Turner, 2011; Abrutyn, 2009; Nolan \& Lenski, 2009) offers the vocabulary and the theoretical tools to supplement the meso-level and social psychological models by precisely conceptualizing the macro-level, especially institutions 
as spheres or domains of social action, delineating the emergent properties and dynamics that shape and are shaped by the meso- and micro-levels of analysis, and removing the vagaries surrounding these "broader" environments and the exogenous shocks. This effort begins with the reader reconceptualizing the social universe as a series of nested hierarchical levels (Turner, 2010). Unlike Parsons' "box-within-a-box" model, a nested hierarchy only refers to the vertical embeddedness of various social phenomena. That is, each level is distinct from other levels because of emergent properties and dynamics, yet there are structural and cultural dimensions which draw these levels together by emanating from the top and from the efforts and habits coming from the bottom.

In particular, this paper will examine the most fundamental building blocks of all human societies: institutions, or macro sociocultural and structural phenomena that organize actors, resources, and authority systems based on the basic human concerns they deal with, the pattern of resource distribution within the institution, and their relationship to other institutional domains. On the one hand, institutions are vital to a comparative-historical sociology because there are a limited set of ubiquitous ones - kinship, polity, religion, law, economy, and education (Turner, 2003; Nolan \& Lenski, 2009) in addition to some recent secondary institutions like medicine, science, sports, and art Abruty, 2009). That there are a finite set allows for analyses that transcend the limitations found in many of the meso-level models that tend to overemphasize the modern, economic corporation or university or nation-state. On the other hand, there are certain institutional dynamics and processes which vary across time and space which lead to very different looking societies and, of course, corporate and individual actors.

For the purposes of the paper below, the most important dynamic that sets institutions apart from each other, in time and space, is their level of autonomy relative to other institutions (Abrutyn, 2009). Autonomy is a process by which certain corporate actors, institutional entrepreneurs (cf. Eisenstadt, 1980; Colomy \& Rhodes, 1994), innovate and struggle for resources; when successful, entrepreneurs secure structural and symbolic independence vis-à-vis other social units. The greater the degree of independence, the greater their ability to (a) reconfigure physical, temporal, symbolic, and social space in meaningful ways that (b) create relatively discrete zones or spheres of institutional action called institutional cores as well as (c) institutional space called institutional environments characterized by the distribution of various non- or extra-entrepreneurial actors either closer or further away from the core. Hence, there are institutional-ecological dynamics premised on the degree of autonomy the institution has, the degree to which the core is physically and cognitively distinct from other cores, and the relative distance an individual, corporate actor, or field finds itself from one core or another. As such, the theoretical effort below offers the outlines of a theory of institutional ecology in hopes of supplementing the insights of meso- and micro-level models by formally elucidating the contours of the institutional core and its environment. First, however, some definitional work is in order.

\section{Theoretical Considerations}

\subsection{Institutions and Their Autonomy}

For macro sociologists, institutions are spheres or orders of social action. They are distinct from other types of social phenomena because they are macro in nature; they cannot be organizations, despite the colloquial use of the term, because they are comprised of multiple corporate units. Although some organizations often resemble institutions in their material and symbolic dimensions, any given organization can only reflect a portion of an institution and its material/symbolic resources (Luhmann, 1984). Thus, institutions are 'warehouses' of elements that individual and corporate actors select from. Some elements are ubiquitous across the corporate units within the domain, whereas others are unevenly distributed.

Institutions are important for a couple of reasons. First, they channel social action. Institutions are built through adaptation to various problems, and reflect the past actions, goals, and decision-making procedures perceived as being successful - regardless of their objective success. As such, institutional domains are organized around one or more human concerns, which are typically intangible, abstract goals. Ultimately, concerns like "justice" or "salvation" become the abstract, underlying purpose(s) or goal(s) of the institutional domain (Parsons, 1990). To be sure, intangible goals are linked to proximate, concrete goals within institutions - e.g., salvation gets linked with ethical prescriptions meant to achieve tangible ends now and grace later. Hence, institutions become spheres of social action where various intermediate means-ends relationships are linked together in action chains. For individual and corporate actors, then, institutions constrain and facilitate action, goal setting, and decision-making by delimiting paths while encasing these paths in authority systems rooted in various mechanisms of control.

Second, while institutions do not act or think, it is not incorrect to suggest institutions are real in physical, 
temporal, symbolic, and social space. Religious institutions, for instance, reconfigure space and time, as well as symbols and social relationships by demarcating the "sacred" from the "profane". Polity does the same, but through different codes and logic, as do economy or kinship. The ways in which an institution is manifest along those four dimensions has varied effects on individuals, corporate actors, and the construction and reproduction of corporate fields or niches.

Third, institutions can and do grow more or less autonomous over time. Institutional autonomy is the process by which key corporate actors (institutional entrepreneurs) monopolize technological, organizational, and/or symbolic knowledge/practices associated with one or more human concerns, become indispensible to a significant proportion of the population, and secure structural and symbolic independence vis-à-vis other social units such that they are able to reconfigure physical, temporal, and symbolic space as well as social relationships in meaningful ways (Abrutyn, 2009). Institutions become more or less autonomous based on the success or failure of institutional entrepreneurs as they innovate to solve certain problems, monopolize these innovations, legitimate their new cultural orientations, and secure power-dependence with other social actors. From a macro perspective, autonomy means physical, temporal, symbolic, and/or social space grows more distinct: political or kinship zones/districts appear as the central locus of entrepreneurial activities, as well as key buildings; specific times like 'holy-days' or "business hours" become separate from profane or "family time"; architecture, emblems, and uniforms demarcate space, time, and role players from each other; and, discrete generalizable social relationships emerge such as doctor-patient, parent-child, or producer-consumer.

On the micro level, autonomy generates increasingly discrete sets of actions, goals, and decision-making processes. For example, in the U.S., where kinship and economy are relatively autonomous institutional domains, there are many familial behaviors clearly distinguishable from economic behaviors: buying stocks or choosing the best deal is very different from participating in family traditions or taking family vacations. Likewise, many economic goals are obviously discrete from kinship as they are often incompatible and even conflicting. Finally, one would expect economic decisions to be made following a particular logic, whereas familial decisions are supposed to be less instrumental and more substantive. This begs the question: why do some kinship actors act, set goals, and make decisions in ways we would expect - e.g., culturally legitimated patterns, while others appear oriented towards other institutional domains and their goals? Why do some political actors make decisions based on morality/piety or money, while others decide based on political expediency or rational logic rooted in the discourse of power?

On the meso-level, we would expect to see organizations which increasingly reflect the institutional structure and culture: economic organizations will be oriented towards the accumulation of the generalized symbolic media of economy, money, while political organizations orient towards power and kinship towards love/loyalty. More interestingly are the ways meso-level models like Fligstein and McAdam's (2011) strategic action fields (SAFs) can be powerfully supplemented. SAFs are characterized by shared understandings regarding "what is going on in the field," by a "shared understanding about the "rules" in the field, and a relatively common "interpretive frame" that orients both corporate and individual actors. Little, however, is said about why and how these three characteristics may vary across SAFs. It is clear that SAFs found in one institutional domain will vary from those found in others; moreover, the greater the level of autonomy of, say, polity or economy, the more discrete the SAFs' rules and interpretive frame. Finally, as we shall see, SAFs can and often do emerge within the interstices of two or more institutional environments, and the consequences are obvious: the rules may blur, innovation may be likely, and conflict may intensify over which "interpretive frame" is the "correct" frame. Thus, institutions are not just composed of organizations and individual role-players, but also the networks, niches, and fields comprised of congeries of actors.

\subsection{Laying the Foundations for A Theory of Institutional Ecology}

As early as Spencer and Durkheim, sociology drew parallels between society and the biotic environment, arguing that populations or segments of the human population were analogous to species in ecological niches: resource scarcity and population pressures drove the types of sociocultural dynamics shaping human societies. In the twentieth century, Amos Hawley (1944) offered a formal theory of human ecology. Interested in population-level dynamics, Hawley argued populations grow based on their ability to adapt to a changing environment. For a population to grow, it needed more physical space; physical growth, though, was governed by available communication/transportation technologies; hence, advancement in these technologies offered populations access to greater niche space and, therefore, physical growth. More recently, Hannan and Freeman (1977) elucidated a theory of organizational ecology that conceptualized populations of organizations as growing, stagnating, and contracting within resource 'niches' (cf. McPherson, 1983). Their work drew more parallels to Durkheimian ecology: organizations faced resource scarcity within a given a niche because of competitors 
producing or distributing similar goods or services; niches could grow larger or segment into new differentiated niches where technological advances occurred or where new divisions of labor emerged. Thus, an organization's 'location' in terms of their access to niche-specific resources affected their survival rate, as well as mobility. Given that institutions are characterized as spheres of social action, it is reasonable to assume they to elicit ecological dynamics.

\subsubsection{Shils' Functionalist-Ecology}

Shils (1975) functionalist-ecological model provides a good foundation for conceptualizing an institutional ecology. Shils argued that every society had a physical "center," comprised of the most central institutions, value-system, and authority system. Besides being a physical location the center was also:

the symbolic or cultural center of a society. And while some of the cultural elements are derived from and shared with other societies, part of this culture will be particular to itself. Some of this particular culture will be about itself. It will consist of beliefs about the history and nature of the society, its relationship to certain ideal or transcendent entities or values, its origin and destiny. It will include beliefs about the rightfulness of its existence as a society and about what qualifies its members to belong to society (1975:36-7, emphasis added).

Shils' theory is a good starting point because he visualizes society in real ecological space: a center and its environment exists. The further an actor is from the center, the weaker the mechanisms of control are and, concomitantly, so is the level of commitment the actor has towards the symbolic system - e.g., legitimated values or norms. However, we must go further than Shils, as much of his work was founded on functionalist fallacies. For example, aside from small, hunter-gatherer societies and chiefdoms, one would be hard pressed to find a large-scale, complex society with a single center. Furthermore, the actual term "center," implies some degree of value-consensus when, in fact, a center may have competing corporate actors with competing visions of reality. Indeed, inherent in his choice of language is the assumption of a single value-system to go along with his single center, when it is more plausible to think of the center as being a warehouse of symbolic elements, some of which are ubiquitously accessed while most others are unevenly distributed by class, gender, or other categoric distinctions; and in many cases, may even be competing or contradictory in content and meaning.

\subsubsection{Autonomy and Ecology}

Abrutyn's (2009) work suggests moving away from the single-center model by shifting the lens of analysis from the society to the institution. Because institutions occupy physical, temporal, symbolic, and social space, increasingly so with greater autonomy vis-à-vis other institutions, it is plausible to suggest each institution develops its own core amidst a surrounding environment that includes other institutional cores as well as corporate units devoted to the core in question. Greater autonomy equals greater physical, temporal symbolic, and social boundedness around the institutional domain's core, which simply means actions and attitudes within the core are less influenced by other institutions. The environments of all institutional domains continue to overlap, reflecting the interrelationship between institutions and, more importantly, the interconnectedness of individual and corporate actors found within the institutional environment.

Where an institutional core has emerged apart from other cores, it can be said that its constituent entrepreneurs have secured greater amounts of independence vis-à-vis other social units and the core is the central locus of the production, distribution, exchange, and consumption of valued institutional resources. These resources may be intangible and substantive (e.g., salvation) or they may be other desired resources like power, prestige, wealth, and so on. Given these conditions, we would expect actors to be effected by the level of institutional autonomy a particular institution has and their relative distance from the core. Taking into consideration Fligstein and McAdam's (2011) strategic action fields, which are arenas of varying duration in which groups struggle for resources, especially power, one could argue that even the fields or niches consisting of congeries of corporate and individual actors will be effected by an institution's autonomy, their distance from the core, and the degree to which they are found within the overlaps of multiple institutions. A general rule of proximity is tentatively proposed: the closer are an actor or set of actors to the core of an institution, the greater is the visibility and access to institutional resources and authority systems and, therefore, the more likely they are to act, set goals, make decisions, and use strategies sanctioned by the institutional domain. The rule of proximity suggests a continuum of predictability in individual and corporate action.

\section{Institutional Space}

\subsection{The Core}

An institutional core contains an institution's central actors, resources, and rules. Cores are the central locus of various types of entrepreneurial activities: some meant to reproduce the institution and its resource base and others to expand the boundaries between itself and other institutions. Like any resource niche or "field," the core 
is also an arena of conflict and struggle. The difference between the core and other intra-institutional sectors is the stakes of the competition: when one faction succeeds in besting another, they can monopolize the production and distribution of resources, propagate their vision of reality as legitimate, and can qualitatively reshape the structure and culture of the entire institution as well as potentially affect other institutional domains. However, as institutions gain greater autonomy, the rules governing competition become rigidified and reconfiguration becomes rarer.

The physical core of an institution may be located in a single place, such as polity being demarcated by a Palace in the capital of the earliest empires. Or, like the American polity, a distinct core such as Washington D.C. may exist, but its distance from the population and the premium on control causes "mini-cores" to emerge in every state capital and major city, as well as in administrative buildings throughout the U.S. These seemingly separate physical sites do not act as cores, but rather as cognitive and/or symbolic reminders and cues used by institutional entrepreneurs for control over distant locations; and while the actors found in these mini-cores are authorized by entrepreneurs, as we shall see below, they act in support roles more so than executive roles. It is also possible for some institutional domains to be decentralized and have multiple cores in different locations or competing within a single physical space. The U.S.'s religious institution is notably pluralist, characterized by every church or synagogue being a physical core. Make no mistake, though, despite myriad disparate cores, religion in the U.S. still constitutes a discrete, relatively autonomous institutional domain that shares some symbolic and material elements across cores. What differs, are the modes of integration between each core or organizational unit: rather than rely on domination or exchange to coordinate resource flows and interaction, these institutional domains use inclusion/embedding mechanisms, mobility, and draw from a limited warehouse of symbolic elements, which produce greater structural and symbolic equivalences (Abrutyn and Turner, 2011).

Finally, in some cases the physical core is nearly impossible to locate and only emerges in temporal, social, and symbolic space. Take, for instance, kinship in modern industrial societies. One could argue the individual household is the core, but it is perhaps more correct to point to "residential zones" or entire communities as the physical core of the kinship domain. The vague borders of an institution like kinship in a complex industrial/post-industrial society, may be best examined in light of how the its core emerges through temporal distinctions (work time versus family time), social relationships (the generalized "parent-child," "husband-wife" role-sets versus employee-employer, consumer-producer role-sets), and symbols (love/loyalty versus money).

All institutions, of course, vary in terms of the level of physical or symbolic discreteness; only empirical analysis can discern the ecological shape of a specific case. In some cases, truly distinct social relationships do not exist because an institution lacks a certain degree of autonomy, while in others, roles are clearly bounded by activities and knowledge-bases; symbols may range from being highly shared between institutions to highly discrete; and some institutional domains may be highly differentiated in symbolic reality, but less so in temporality. However, as a general rule, it is proposed that the greater is the degree of institutional autonomy, the greater is the degree to which a given institutional core is physically, temporally, symbolically, and/or socially discrete vis-à-vis other social spheres. And, with greater discreteness comes greater distinction in role-performance among entrepreneurs as well as the meanings, expectations, and obligations of all social relationships that are realized within the core.

\subsection{The Institutional Environment}

The environment, like the core, is also distinguishable in physical, temporal, social, and symbolic space, but it is the way groups are distributed within this space that is of interest. Five general groups are identified below: (1) support, (2) liaison, (3) consumers, (4) extra-institutional entrepreneurs, and (5) oppositional actors.

\subsubsection{Support Actors}

Not every person trained in the core of an institution comes to occupy an entrepreneurial position. They are imbued with the some degree of authority derived from the core, but by choice or by external social conditions find themselves in positions further from the core. In the ideal, their primary function is in support of entrepreneurs - e.g., the bureaucracy of an autonomous polity. Their everyday activities are invaluable to the reproduction of the institutional core as well as boundary maintenance, but they are less privileged and lower in status than those in the entrepreneurial unit. In a sense, they are middle managers whose ideological commitment is often very high because they derive relatively high rewards and are therefore often 'in debt' to entrepreneurs. Part of the pressure to conform derives from their relationship to consumers or extra-institutional actors. In many cases, they are the face or representatives of the institution, as those in the core are often removed from the mundane aspects of institutional life. Being considered the representative puts pressure on people to act in stereotyped ways, for fear of losing face, not being considered authentic, or tarnishing the institution's perceived 
reputation. And while it is always possible that challenges to the core and its entrepreneurs may come from this sector, it is unlikely as these actors have the most to gain from institutional stability.

\subsubsection{Liaison Actors}

Liaison actors are a special case of support actors, but their overall commitment and level of predictability is often much lower. They are found in the interstices of two or more autonomous institutional environments in positions akin to Burt's (1992) structural holes. Their primary function is to translate the symbolic code of the institution they belong to into a language the consumer or extra-institutional entrepreneur can understand, translate the symbolic language of other institutional domains into a language the entrepreneurs they serve can understand, and, ultimately, facilitate consumer or extra-institutional entrepreneurial access to their institutional domain's core. They serve as the link between the core, access to whatever specific resources or general concerns exist within in it, and those who desire these resources or concerns. Because they find themselves dealing with both elites of one or more institutions as well as clients who provide them with much of their material rewards, they sometimes have conflicting loyalties, commitments, and behavioral/attitudinal dimensions. Additionally, they are often indispensible to institutional exchanges, which gives them leverage - e.g., their command over multiple institutional symbolic codes allows them to manipulate other actors as does the fact that the interstices between institutional domains are often ambiguously defined and weakly monitored and sanctioned affording liaison actors latitude. Often times, liaison actors become entrepreneurs themselves, carving out new autonomous space for other institutions. While other times they may act as the most powerful source of reformative change, as they work within the system, represent the positions of various types of actors, and still have the unique role of accessing the core and its entrepreneurs.

\subsubsection{Consumers}

Calling those pursuing what the institutional core offers consumers may appear economically biased, but it is a fitting analogy for those more specific roles like "client" or "patient." It is the most accessible and generalized role in any autonomous institutional domain because it has simple membership criteria, even if access may be restricted by categoric distinction. Consumer are vital to sustaining an institution's autonomy: the greater is the proportion of the population willing and able to be a consumer, the greater the diversity of resources available to an entrepreneurial unit; a more diverse resource base means less dependence on a single source and, therefore, greater structural/symbolic independence.

For example, a person may never go to court ever or perhaps only once. Despite this fact, most adults within a society with a relatively autonomous legal institution understand what it means to be a "client" and, to some extent, know what to expect when acting within the legal institution. Consumers can be latently oriented towards an institution's core because it becomes "normal" for most people to assume that justice is obtained in the legal institution, which offers the only "sanctioned" or "legitimate" mechanisms of conflict resolution.

\subsubsection{Extra-Institutional Entrepreneurs}

This type of actor poses both a threat and safeguard to an autonomous institution's core and its own entrepreneur's independence. On the one hand, entrepreneurs depend on each other as both consumers as well as sources of legitimacy - e.g., political entrepreneurs in the U.S. depend on a relatively autonomous legal institution for their own authority, while legal entrepreneurs need power, as franchised authority from the polity, to enforce their decisions and make them binding. Often, entrepreneurs share similar class positions, life chances, and opportunities, which can lead to status-group formation within an elite network. On the other hand, entrepreneurs are always striving to expand the influence of their core, often at the expense of other autonomous institutional domains. Thus, where the first states emerged in Mesopotamia, Egypt, or China, polity and kinship existed in a tenuous relationship though one which typically saw their respective entrepreneurs at least bolstering each other's claims to legitimacy (Abrutyn and Lawrence, 2010). This relationship was characterized by efforts to sustain and expand each party's respective institution's autonomy. Polity, which is the central locus of the symbolic medium of power and which is backed by the legitimate use of force, has been far more successful in eroding kinship entrepreneur's efficacy.

\subsubsection{Oppositional Actor}

This broad category accounts for every type of actor who finds themselves on the margins of an institution or set of institutions; and may include extra-societal actors. The goals of oppositional actors vary, ranging from a desire to be included in the core and have access to some of the positions to wanting to tear the very core down and do away with the institution's autonomy. More often than not, the intensity of their goals is predicated on their relative distance to the core. Those near enough likely derive some reward and are not interested in destroying 
the very source of their wealth, power, prestige, and so on; their desire is likely for more access. Those further from the core or in the interstices of overlapping institutional environments likely want to usurp one core in the name of another core, or they want to merge the two in a way that would produce a new structural reality. Finally, those on the margins are either denied access to the core or choose not to have access to the core and tend to be revolutionary in nature. Thus, boundary overlaps produce interstices and, often, structural. Innovation of all sorts often happens in the overlaps or on the margins as social movements emerge combining elements of one institutional domain with another are more likely to occur. In addition, individual and corporate actors may find diversifying their commitment produces a greater amount of reward than had they committed to a single institutional core.

Finally, at the physical and cognitive margins of every autonomous institutional environment stand the strangest amalgamation of categoric units: the underclass, the exploited, the retreatists, the enemies of the state, the mentally insane, and other similar groups. In most cases they are 'deviant' because they follow unpredictable paths in ways that disregard or challenge the dominant norms and values. In some cases, they are oppositional actors while in others, such as the case of the mentally insane, they are simply unstable actors (in terms of societal norms) whose presence may be benign or harmful. In essence, the margins are both the loci of potential resistance or revolutionary movements, as well as ascetics, deviants, and other fascinating outliers.

\section{Ecological Dynamics}

\subsection{The Rule of Proximity}

As institutions become more autonomous, it is expected that institutionalized action, goals, and decision-making processes grow more discrete within the core and the environment. Recall the rule of proximity: the closer are an actor or set of actors to the core of an institution, the greater is the visibility and access to institutional resources and authority systems and, therefore, the more likely they are to act, set goals, make decisions, and use strategies sanctioned by the institutional domain. Distance can be measured in two interrelated ways: physically and cognitively. Actors may be physically close to the core, but not cognitively oriented towards the symbolic elements of the institution; conversely, people may find themselves far from a core, while cognitively close. That is, a judge may be sitting on her bench, but be oriented towards religious or political symbols in ways that other judges - who are more highly committed to an autonomous legal system - are not. Conversely, a judge may take a vacation or go and vote and carry their judicial identity with them in ways affecting their behavior in these non-legal domains. It is posited that where actors are both physically and cognitively close, predictability in action, goals, and decisions should be highest. Where people are physically close and cognitively far, or vice versa, predictability will be governed by situational and structural factors. Finally, as both physical and cognitive distance increases, predictability decreases. Thus, our first proposition can be posited:

P1: The degree to which an individual or corporate actor's behavior and attitudes will be predictable and conform to institutional standards is a positive function of (a) their physical proximity to the core and (b) their cognitive proximity to the core.

\subsection{Physical Proximity}

Institutions are real things because they occupy real physical space - that is, buildings, role players, and other external manifestations exist in geographic space. These social objects serve as reminders of the institutional system, its entrepreneurial unit, its real or perceived function(s), and its meaning complex, while having concrete functions. Not every actor is socialized equally, and as such, some actors will be more cognitively in-tune with these representations than others. Nevertheless, it is safe to say that any actor who is socialized within a group that finds itself within a culturally, politically, and territorially bounded system will be aware of and primed for understanding and orienting towards these physical cues. Four mechanisms seem to undergird the dynamics of physical proximity: the extensivity of networks ties; the level of formality; the visibility of tangible resources; and the visibility of mechanisms of social control.

\subsubsection{Extensive Network Ties}

Sheldon Stryker (1980) posited that as the number of people who know a person and interact with him or her in ways that activate one of his or her identities goes up, the level of extensive commitment will rise concomitantly. That is, a role which is routinely and frequently activated, vis-à-vis any other role he/she may play, is likely to be more salient and prominent. Furthermore, the actor is likely to derive greater extrinsic rewards (and perhaps intrinsic rewards) in roles that are frequently activated; the punishments for inappropriate behavior will be more obvious and their consequences more problematic. To be sure, corporate actors found in dense niches, cooperating and competing against extensive networks of other similar corporate actors will face pressures to 
conform and look and act appropriately.

It seems logical to suggest as an actor or set of actors get closer to an institution's core the frequency with which (1) institutional roles or corporate functions are activated, (2) role-performance or organizational compliance and conformity is expected, and (3) actions and goals are rewarded or punished should increase. Support and entrepreneurial actors, especially_because they frequently interact with entrepreneurs - tend to be strongly committed to the institutional domain, are likely to conform to expectations, and are also likely to ideally represent the institutional domain beyond its physical boundaries. Extensive commitment is simply a function of their daily rounds. Those located further away will be less likely to interact with institutional actors frequently, and therefore, less likely to have that identity activated and rewarded.

Others, such as consumers are 'guests' of the institution, and commitment is temporally based because there is little extensivity beyond their ephemeral encounters. In these cases, it is the nearness to the mechanisms of control that are more important for conformity than the extensivity of networks ties. For example, a visitor to a church or synagogue will look around and gather data as quickly as possible regarding how to act appropriately in order to prevent embarrassment, disruption of activities, or other potentially harmful sanctions. The larger the crowd and the denser their distribution, the greater is the force exerted on outsiders for conformity. Moreover, greater numbers of people make outsiders aware of their performance such that they attempt to maintain a consistent performance for the duration of the situation, if only to demonstrate one's competence at playing roles. In either case, the extensivity of network ties and, therefore, role commitment is positively related to physical proximity. It is posited:

P2a: The closer an actor, corporate unit, or field is to the core, the greater the extensivity of network ties and, therefore, the greater is the likelihood that (a) the individual or corporate actor will act, set goals, and make decisions in predictable, institutionally-specific ways and/or (b) the field of actors will adopt institutionally-specific rules and interpretive frames, and be governed by and pursue institutionally defined resources.

\subsubsection{Formalization of Situations}

The physical space within the core and the closest physical regions surrounding the core are typically formalized: ecological arrangements, temporality, power-relations, interaction rituals, and other situational elements are embedded in the arrangement of space constraining action. To be sure, random and unpredictable things occur because humans do not always act in ways we would expect, but formal settings offer very clear cues to actors about what is expected in terms of emotion (Collins, 2004) and impression management (Goffman, 1959).

Put another way, greater situational certainty induces stronger levels of trust in and commitment to other actors and one's role performance. A person familiar with being a consumer in one institutional-setting will be comfortable as a consumer in a novel setting. Hence, high levels of certainty produced by settings being formal reduces the level of ambiguity associated with impersonal interactions as expectations and obligations are relatively known to both the novice and the gate keepers. Of course, formalization decreases with physical distance from the core. A professor may refer to themselves as "Dr." outside of the classroom at a bar or supermarket, but it is not guaranteed that the 'other' will treat them with the deference he or she receives in their formal milieu (e.g., the classroom). Thus, it is proposed:

P2b: The closer an actor, corporate unit, or field is to the core, the greater is the degree of formalization as found in the physical arrangement of space, and, therefore, the greater is the likelihood that (a) the individual or corporate actor will act, set goals, and make decisions in predictable, institutionally-specific ways and/or (b) the field of actors will adopt institutionally-specific rules and interpretive frames, and be governed by and pursue institutionally defined resources.

\subsubsection{Resource Visibility}

The closer one gets to the core, the more visible desirable resources are. Sociology typically recognizes power, prestige, and wealth as key, but the core also offers other valued resources-verification, gratitude, affection/attention, and so on (Turner 2002). In addition to these general social resources, the core produces and distributes institutionally-specific resources. While entrepreneurs directly benefit from monopolizing the productive process, they are not the only actors who benefit from the core. The core, for instance, can produce strong collective emotions. Being emotional creatures, many people are drawn to these sites to gain access to them. As one gets physically closer to the core, these things become visible such that they 'whet the appetite' and orient individual and corporate actors to figure out the terms of exchange, the path(s) of mobility, and secrets of entrepreneurship. Conversely, being further away means some resources are obfuscated by distance alone. The 
outlines of what power or prestige are can be seen in the distance, but the reality of these resources and other institutional resources is hidden or too far from one's everyday reality to control their orientations. It is also possible that distance weakens commitment as other institutional domains offer resources which compete and lead to the activation of very different roles.

P2c: The closer an actor, corporate unit, or field is to the core, the greater is the degree to which desired resources are visible, and, therefore, the greater is the likelihood that (a) the individual or corporate actor will act, set goals, and make decisions in predictable, institutionally-specific ways and/or (b) the field of actors will adopt institutionally-specific rules and interpretive frames, and be governed by and pursue institutionally defined resources.

\subsubsection{Social Control}

By mechanisms of social control, we refer to the entire system of authority which includes agents who can monitor behavior, explicit and implicit rules dictating expectations and obligations, and finally, sanctions for appropriate or inappropriate behavior. For now, we are referring to the external, regulatory/coercive forms of control. The closer one gets to the physical location of the core, the greater the degree of social control exerted on them. We noted already the force exerted by larger numbers of institutional actors being present in a denser amalgamation; the likelihood that a non-sanctioning agent may sanction inappropriate action increases with closeness to the core. Furthermore, as one gets near the core or enters the core, individuals or corporate actors specifically authorized to monitor and sanction will become more visible and present. And because monitoring and sanctioning costs can be very expensive, it is likely that entrepreneurs will expend resources on external mechanisms close to the core where the uninterrupted production, distribution, and consumption of institutional resources is desired by entrepreneurs as well as support, liaison, and consumptive actors. It is in the interests of those in the core to ensure that their activities are not interrupted, and where institutions have become highly autonomous, they will have a distinct authority system in place to 'coerce' compliance. Thus, we posit our final proposition associated with physical proximity:

P2d: The closer an actor, corporate unit, or field is to the core, the greater is the degree to which mechanisms of social control are visible and less costly, and, therefore, the greater is the likelihood that (a) the individual or corporate actor will act, set goals, and make decisions in predictable, institutionallyspecific ways and/or (b) the field of actors will adopt institutionally-specific rules and interpretive frames, and be governed by and pursue institutionally defined resources.

The four dimensions can be summarized:

P2: The effects of Physical Proximity on an individual or corporate actor, or the field in which they are embedded, is a positive function of: (a) the level of extensive ties, (b) the degree to which institutional situations are pre-defined, certain, and formalized, (c) the visibility of desired or desirable resources, and

(d) the degree to which external mechanisms of control are visible and salient.These four variables are independent of each other, but function additively on producing greater or lesser predictability in individual and corporate action, goal setting, and decision-making.

\subsection{Cognitive Proximity}

If people can be physically closer or further from the institutional core, they certainly can be cognitively closer or further away. That is, a government is only real and legitimate if a significant proportion of people believe it to be real and legitimate by orienting their actions and attitudes accordingly. To be sure, an illegitimate government can act in ways that are real, such as squelch a rebellion, oppress its subjects, or wantonly use violence. But, the use of naked force is costly and eventually it sows the seeds for resistance, revolution, or collapse. Hence, governments, like any group with some centralized power, seek to legitimize their visions of reality, their structural positions, and their symbolic systems such that role-players internalize them, take them for granted, and routinely act in appropriate ways. Furthermore, if an entrepreneurial unit can sanctify their vision of reality, acting "correctly" and setting some goals over others can become moral imperatives. Three particular dimensions related to cognitive proximity seem to generate greater levels of role or organizational predictability: the intensivity of network ties; the degree of access to symbolic capital; and the degree to which an actor has internalized the mechanisms of control.

\subsubsection{Intensive Role Commitment}

A role will be more salient and prominent, and therefore more prone to high levels of commitment, if the others that frequently activate it are significant others (Stryker, 1980). Commitment is predicated on the strength of and reward from the emotional exchange with significant others (Lawler, 2006), as well as the shared history and 
multiplex nature of the exchange relationship. Additionally, Burke (1991) has argued that significant other's evaluation and verification of our identity is the cornerstone to role performance and interaction in general as lack of verification generates negative affect (cf. Stets, Carter, Harrod, Cervin, and Abrutyn, 2008). Where institutions are autonomous and their roles clearly defined in relationship to other types of roles, it becomes possible for an individual to be known or thought of by their significant others as that role.

Again, corporate actors do not "commit" to roles, but they can be situated in more advantageous locations in an institutional domain which leads to a particular cultural pattern that emphasizes the prestige of being a member. Yale or Harvard are generally considered at the center of the higher educational system, and as such new or aspiring members already are aware of this and through the socialization process of becoming faculty or an administrator these premises are further reinforced. The corporate unit, when filled with individual actors perceiving their status as high and recognizing it derives from the organization's location, does indeed act in ways representative of its position and sets goals commensurate with the educational core. It is proposed:

P3a: The closer an actor, corporate unit, or field is to the core, the greater the intensivityof network ties and, therefore, the greater is the likelihood that (a) the individual or corporate actor will act, set goals, and make decisions in predictable, institutionally-specific ways and/or (b) the field of actors will adopt institutionally-specific rules and interpretive frames, and be governed by and pursue institutionally defined resources.

\subsubsection{Access to Symbolic Capital}

Perhaps no dimension is more important to cognitive proximity than how much access a role player has to the most valued symbolic resources, their development, application, dissemination, and consumption. Autonomous institutional cores become the storehouses, the factories, and the markets for the use of certain resources in particular: generalized symbolic media of exchange (Abrutyn, 2009). Symbolic resources are not quite like tangible things like status goods because the pursuit of generalized symbolic media of exchange such as love/loyalty, power, or money implies the internalization of a whole set of values, ideologies, beliefs, and norms associated with obtaining, storing, investing, and using these symbolic resources (Abrutyn and Turner, 2011). Symbolic media, then, are bundles of institutional codes, motives, justifications, ideologies, and strategies; they are resources that can be saved, invested, transferred across boundaries for other institutional media of exchange; and they can be hoarded by some groups over others.

Where persons or corporate units are oriented towards getting more of a particular institution's medium they are likely to be committed to the prescribed paths to resource attainment-e.g., their choices will be somewhat circumscribed, regardless of available alternatives. Additionally, the more they desire these resources, the more they internalize particular institutionally-specific symbolic elements. Thus, a professor who seeks knowledge and applied truth, an artist who pursues beauty, a religious actor seeking piety/morality, or a kinship actor seeking love/loyalty will pursue these resources in relatively predictable, prescribed patterns as defined by the broader institution, respectively. Subsequently, the greater is an actor's access to a symbolic medium of exchange, the greater is likelihood with which he/she has internalized the norms, values, and ideologies associated with the medium. Therefore, the more they internalize these institutional elements the more in line their actions, goals, and decisions will be with institutionally specified actions, goals, and decisions.

Again, corporate actors are like individual actors: they do orient their goals and actions towards accumulating generalized media of exchange. However, unlike individuals, specific corporate units-e.g., institutional entrepreneurs - can monopolize the production and distribution of media within a relatively autonomous institution, and those in privileged positions can become storehouses of the media as well as market places for the investment and transference of one medium for another. Our second proposition concerning cognitive proximity can be stated:

P3b: The closer an actor, corporate unit, or field is to the core, the greater is the degree to which an actor can see, pursue, obtain, and use institutionally-specific symbolic resources, and, therefore, the greater is the likelihood that (a) the individual or corporate actor will act, set goals, and make decisions in predictable, institutionally-specific ways and/or (b) the field of actors will adopt institutionally-specific rules and interpretive frames, and be governed by and pursue institutionally defined resources.

\subsubsection{Internalized Mechanisms of Control}

Some mechanisms of control are purely external to the actor, while others are instilled through socialization. Cultural-cognitive mechanisms are those rules, sanctions, and authority figures which become taken for granted aspects of the everyday life of the individual, while normative mechanisms become imbued with ethical and/or 
moral imperatives compelling 'voluntary' action.

Greater internalization means a number of different things. For institutional entrepreneurs it means reduced monitoring costs because it implies conformity beyond the physical boundaries of an institution is more probable: Role-players carry the norms and values of the roles they most identify with, or more correctly, roles act as the vehicle of social structure. Some of these norms and values translate across situations and institutional boundaries, while others are institutionally-specific and can cause some disruptions in non-institutional encounters. Either way, the internalization of the "rules of the game" carries consequences for action and attitudes. The interpretation of societal events or other's motives, the interaction strategies and binding themes of discourse within an encounter, and the justification an actor uses for their behavior all are tied to how constrained they are by the institutional rules they have internalized. Our final proposition concerning cognitive proximity can now be posited:

P3c: The closer an actor, corporate unit, or field is to the core, the greater is the degree to which normative or taken for granted mechanisms of social control are internalized and enforced, and, therefore, the greater is the likelihood that (a) the individual or corporate actor will act, set goals, and make decisions in predictable, institutionally-specific ways and/or (b) the field of actors will adopt institutionally-specific rules and interpretive frames, and be governed by and pursue institutionally defined resources.

\subsection{Summary}

Cognitive proximity can tell us a great deal about an actor's orientation to structure and culture, allowing us to explain why some actors organize their goals and actions in stereotyped ways while others are labeled 'erratic', 'corrupt', self-serving, or whatever other labels are adopted to define deviance. Those who find themselves close to the core are likely to exhibit strong tendencies towards patterned action and goal-setting, while those further from the core will vary in their tendencies. We can finally offer a general proposition concerning cognitive proximity before examining this exception to the rule of proximity:

P3: Cognitive proximity is a positive function of (a) the level of intensive ties, (b) the degree to which an actor can see, pursue, obtain, and use institutionally-specific symbolic resources, and (c) the degree to which normative and/or taken for granted mechanisms of social control are internalized and enforced.

\section{Discussion}

Institutional domains like religion or polity are manifest in real physical, temporal, symbolic, and social space. As such, they are sociocultural and structural environments in which individuals, corporate units, and fields or niches comprised of myriad corporate and individual actors are embedded. Thus, a theory of institutional ecology was built above to delineate the emergent dynamics on the macro-institutional level. In particular, institutions grow more or less autonomous as entrepreneurs struggle for resources, position, and structural and/or symbolic independence vis-à-vis each other as well as other social units. Greater autonomy intensifies the ecological dynamics because an institution becomes an increasingly discrete sphere of social action, with a core (or set of cores) that produces and distributes some material and symbolic resources specific to the domain itself and its entrepreneurs and also coordinates and regulates action through institutionally-specific authority systems. The institutional core, then, becomes a physical and cognitive center; and the rule of proximity predicts that the closer are an actor or set of actors to the core of an institution, the greater is the visibility and access to institutional resources and authority systems and, therefore, the more likely they are to act, set goals, make decisions, and use strategies sanctioned by the institutional domain.

More specifically, it was proposed that physical proximity as a mechanism producing greater predictability in action and attitudes is a positive function of (1) the extensivity of institutional ties, (2) the level of formalization, (3) the visibility of institutionally-specific resources, and (4) the degree to which external mechanisms of control were visible and close; cognitive proximity is a positive function of (1) the intensivity of institutional ties, (2) the visibility of symbolic resources, especially generalized symbolic media of exchange, and (3) the degree to which normative and/or cultural-cognitive mechanisms of control were internalized and enforced. Each dimension is independent of the others and should be measured in degree and not kind. And, while it is often the case that physical and cognitive proximity overlap, it is also possible that any given actor or field is physically close to the institutional domain, yet cognitively far - or vice versa.

\subsection{Institutional-Evolution}

The theory above is an important addition to the institutional-evolution tradition. Premised on the Old Institutionalism (for review, see Abrutyn and Turner, 2011), institutional-evolution sees the social world as a series of nested systems (Luhmann, 1984) or hierarchical levels of analysis (Turner, 2010). Institutions are 
considered the basic building blocks of human societies, and six in particular are considered ubiquitous systems or spheres of action - kinship, polity, economy, religion, law, and education (Turner, 2003; Nolan and Lenski, 2009). They are macro structural and cultural domains reflective of the past adaptive efforts of individuals and groups, and have emergent properties distinct from other types of social units like corporate actors. Abrutyn and Turner see institutions as real things whose dynamics are worth understanding because they can supplement the various sociological theories focused on meso- and micro-level dynamics. Hence, a theory of institutional ecology adds to this tradition by explicitly linking the macro to these other levels, suggesting ways in which institutional dynamics like autonomy affect the actions, goals, and strategies individuals or corporate units employ.

\subsection{Fields, Niches, and Networks}

In recent years, some of the most interesting work in sociology has come from organizational sociology. In particular, various concepts denoting the 'environment' in which modern organizations contend with other organizations and uncertainty have been posited: fields (DiMaggio \& Powell, 1983), niches (Hannan and Freeman, 1977), and markets (Fligstein, 1996). Recognizing the limitations of these concepts, Fligstein and McAdam (2011) recently offered a more dynamic meso-level theory of strategic action fields, which integrates the previous models with work on social movements. Moreover, Fligstein and McAdam critiqued the older models as ignoring the environments in which fields or niches were found, adding exogenous forces they termed "broader field environments" and "external shocks."

While an important advance in the meso-level literature, Fligstein and McAdam's model can benefit from the discussion above because it offers greater distinctions in understanding variation across fields and explains where these variations come from. For example, Fligstein and McAdam (2011:4) argue that a strategic action field is characterized by its actors having a shared conception of what the field is about as well as the rules of the field, and actors employ a common 'interpretative frame'. That fields are located in institutions and some institutions are autonomous means that fields also are affected by the rule of proximity. Hence, variation in rules or interpretive schema comes from institutional dynamics shaping the contours of the strategic action field: which institutional domain does it exist or emerge within, how autonomous is the domain in question, how far or near to the core is the field, and so on. Other questions worth examining to further elucidate the link between the macro- and meso-levels include: how are fields in cores different from those in the environment, what happens when a field emerges in the interstices between institutional domains, and how can fields alter the structural and cultural dimensions of an institutional domain?

Moreover, empirical analyses of social movements could be supplemented with an institutional-ecological framework. Consider the most powerful and prevalent anti-systemtic social movements across the globe today - fundamentalist religious movements (Almond, Appleby, \& Sivan, 2003). A cursory analysis would reveal that these movements emerge within the overlap of religion and kinship, which explains why its movement leaders frame non-kin/religious events with a religio-kinship interpretive schema. It would also begin to explain why the masses are drawn to these movements as average people find themselves far from the political and economic cores and therefore derive few rewards from these institutional domains beyond, perhaps, protection from external threats and goods and services in the marketplace. Conversely, kinship and religious institutions are readily accessible and, because of most world religions offering democratized status for all members of the moral community, institutional resources are visible and easily obtainable.

\subsection{Linking the Macro and the Micro}

For the sake of brevity, the links between the macro- and micro-levels can only be briefly touched on. Real generally, however, it is clear that an individual's location within an institutional domain relative to its core and, equally important, other institutional cores will have effects on their self-conception, identity construction, role-performance, and global/institutionally-specific status positions. In essence, this model does nothing to supplant the insights of micro sociology, but rather it supplements it by detailing the structural forces shaping key micro dynamics important to human nature and social interaction (Turner, 2002). For instance, one empirical direction can be suggested: a survey instrument that aims to capture the "role-complex" of an individual actor, or the hierarchy of roles he/she plays, along with demographic and other structural variables could test some of the propositions above in hopes of improving the theory. A judge, for instance, who scores high on numerous dimensions of physical and cognitive proximity would presumably be oriented towards legal norms and values, orient her actions/attitudes towards them, and, perhaps, seek out interactions in which her 'judge' role was activated and salient. Conversely, a judge who spent more time and was rewarded more for being a father may shy away from their judge role, find less satisfaction in working, and may even shirk his responsibilities. 


\section{Acknowledgments}

I would like to thank Randall Collins, Paul Froese, Kirk Lawrence, Isaac Ariail Reed, and Jonathan Turner for comments and suggestions on previous drafts.

\section{References}

Abrutyn, S. (2009). Towards a General Theory of Institutional Autonomy. Sociological Theory, 27(4), 449-465. http://dx.doi.org/10.1111/j.1467-9558.2009.01358.x

Abrutyn, S., \& Lawrence, K. (2010). From Chiefdoms to States: Toward an Integrative Theory of the Evolution of Polity. Sociological Perspectives, 53(3), 419-442. http://dx.doi.org/10.1525/sop.2010.53.3.419

Abrutyn, S., \& Turner, J. H. (2011). The Old Institutionalism Meets the New Institutionalism. Sociological Perspectives, 54(3), 283-306. http://dx.doi.org/10.1525/sop.2011.54.3.283

Almond, G. A., Appleby, R. S., \& Emmanuel, S. (2003). Strong Religion: The Rise of Fundamentalism Around the World. Chicago: University of Chicago Press.

Bourdieu, P. (1977). Outline of a Theory of Practice. Cambridge: Cambridge University Press.

Burke, P. J. (1991). Identity Processes and Social Stress. American Sociological Review, 56(6), 836-849. http://dx.doi.org/10.2307/2096259

Burt, R. S. (1992). Structural Holes: The Social Structure of Competition. Cambridge, MA: Harvard University Press.

Collins, R. (2004). Interaction Ritual Chains. Princeton: Princeton University Press.

Colomy, P., \& Rhoades, G. (1994). Toward a Micro Corrective of Structural Differentiation Theory. Sociological Perspectives, 37(4), 547-583. http://dx.doi.org/10.2307/1389279

DiMaggio, P., \& Powell, W. W. (1983). The Iron Cage Revisited: Institutional Isomorphism and Collective Rationality in Organizational Fields. American Sociological Review, 48(2), 147-160. http://dx.doi.org/10.2307/2095101

Eisenstadt, S. N. (1980). Cultural Orientations, Institutional Entrepreneurs, and Social Change: Comparative Analysis of Traditional Civilizations. American Journal of Sociology, 85(4), 840-869. http://dx.doi.org/10.1086/227091

Fligstein, N. (1996). Markets As Politics: A Political-Cultural Approach to Market Institutions. American Sociological Review, 61(4), 656-673. http://dx.doi.org/10.2307/2096398

Fligstein, N., \& McAdam, D. (2011). Toward a General Theory of Strategic Action Fields. Sociological Theory, 29(1), 1-27.

Goffman, E. (1959). The Presentation of Self in Everyday Life. New York: Anchor Books.

Hannan, M. T., \& Freeman, J. (1977). The Population Ecology of Organizations. American Journal of Sociology, 82(5), 929-964. http://dx.doi.org/10.1086/226424

Hawley, A. H. (1944). Ecology and Human Ecology. Social Forces, 22(4), 398-405. http://dx.doi.org/10.2307/2571805

Lawler, E. J. (2006). The Affect Theory of Social Exchange. In P. J. Burke, Contemporary Social Psychological Theories (pp. 248-267). Stanford: Stanford University Press.

Luhmann, N. (1984). Social Systems. Stanford: Stanford University Press.

McPherson, J. M. (1983). An Ecology of Affiliation. American Sociological Review, 48(4), 519-532. http://dx.doi.org/10.2307/2117719

Nolan, P., \& Lenski, G. (2009). Human Societies: An Introduction to Macrosociology. Boulder, CO: Paradigm.

Parsons, T. (1951). The Social System. Glencoe, Ill.: Free Press.

Parsons, T. (1990). Prolegomena to a Theory of Social Institutions. American Sociological Review, 55(3), 319-333. http://dx.doi.org/10.2307/2095758

Robinson, D. T. \& Smith-Lovin, L. (2006). Affect Control Theory. In P. J. Burke, Contemporary Social Psychological Theories (pp. 137-164). Stanford: Stanford University Press.

Shils, E. A. (1975). Center and Periphery: Essays in Macrosociology. Chicago: University of Chicago Press. 
Stets, J. E. (2006). Identity Theory. In P. J. Burke, Contemporary Social Psychological Theories. Stanford: Stanford University Press.

Stets, J., Carter, M. J., Harrod, M. M., Cerven, C., \& Abrutyn, S. (2008). The Moral Identity, Moral Emotions, and the Normative Order. In D. T. Robinson, \& J. Clay-Warner, Social Structure and Emotion (pp. 227-255). San Diego: Elsevier.

Stryker, S. (1980). Symbolic Interactionism: A Social Structural Version. Menlo Park, CA: The Benjamin Cummings Publishing Company.

Turner, J. H. (2002). Face to Face: Toward a Sociological Theory of Interpersonal Behavior. Stanford: Stanford University Press.

Turner, J. H. (2003). Human Institutions: A Theory of Societal Evolution. Lanham: Bowman \& Littlefield Publishers, Inc.

Turner, J. H. (2010). Theoretical Principles of Sociology: Macrosociology (Vol. 1). New York: Springer. 